\title{
Application of Pre-stranded Tensile Clamp for Aluminum Composite Carbon Fiber Core
}

\author{
Yang Changlong $^{1 *}$, Zhang Hongzhi ${ }^{1}$, Wang Wei ${ }^{1}$, Li Yueyue ${ }^{1}$, Han Rongzhuan ${ }^{2}$ and Dong Qiang ${ }^{2}$ \\ ${ }^{1}$ State Grid Shenyang Electric Power Supply Company, Shenyang, Liaoning Province, 110811, China \\ ${ }^{2}$ Shijiazhuang Huaneng Electric Co., Ltd., Shijiazhuang, Hebei Province, 050011, China
}

\begin{abstract}
The growth of electricity demand has put forward higher requirements for transmission line performance. Aluminum composite carbon fiber cores (ACCC) has better performance than traditional steel core aluminum conductor and can better meet the requirements of power transmission and transformation field. However, at present, the wedge-shaped self-locking tension clamp is usually used for aluminum composite carbon fiber cores, which is complicated in construction technology and poor in economy, which restricts the promotion and application of aluminum composite carbon fiber cores.In order to solve the above problems, three kinds of tension clamp structures applied to different specifications of aluminum conductors composite core are optimized. Through the strength and grip test of the clamp, it is verified that the grip force of the clamp structure designed can reach $95 \%$ of the rated tensile strength of the conductor. Finally, through the comparative analysis of construction and installation, it is concluded that the pretwisted tension wire clamp designed has a good economic effect.
\end{abstract}

\section{Introduction}

Aluminum composite carbon fiber cores is a new type of conductor for the transmission line. It has the remarkable characteristics of high strength, light weight, high corrosion resistance and large carrying capacity per unit area. State Grid Corporation has included carbon fiber composite core conductor in the catalogue of key new technologies[1]. However, with the expansion of the application scale, the problems of aluminum composite carbon fiber cores are gradually exposed. Tensionresistant jointing fittings have become a major bottleneck restricting the promotion and use of aluminum composite carbon fiber cores[2].

In recent years, experts and scholars at home and abroad have systematically studied the practical tension clamp of carbon fiber composite core conductor. Literature [3] analyses the application of pre-stranded tension clamp in transmission line. Literature [4] optimizes the tension clamp structure to solve the problem of overheating. Literature [5] analyzed the factors causing the fracture of the tension clamp of aluminum composite carbon fiber cores, and put forward technical suggestions on the installation and operation of the tension clamp. In summary, there are few studies on the tension clamp of aluminum composite carbon fiber cores. The relevant studies only focus on the structure of the tension clamp, but not on the comprehensive analysis of construction technology and technical economy. Therefore, it is urgent to develop the key technologies of manufacturing and engineering application of new type of pre-twisted metal tools for aluminum composite carbon fiber cores, in order to solve the problems of complex construction technology and technical economy of the metal tools for aluminum composite carbon fiber cores.

In this paper, the structure of pre-stranded tension clamp for carbon fiber composite core conductor is analyzed, three kinds of pre-stranded tension clamps with different structures are designed, and grip force experiment and technical and economic analysis are carried out for the type JLRX/T-450/50 tension clamp for carbon fiber composite core conductor.

\section{Design of Pre-twisted Tension Clamp for Aluminum Composite Carbon Fiber Cores}

Tensile clamp is one of the main fittings for aluminum composite carbon fiber cores. The current wedge-shaped compression tension clamp has the disadvantages of high cost, difficult installation and easy to damage wire core[6]. It is necessary to design and analyze the structure of the tension clamp.

\subsection{Structure and Force Analysis of Pre- stranded Tensile Clamp}

The main factors affecting the performance of prestranded fittings are the ratio of inner diameter of helix to outer diameter of conductor (ID/OD), diameter of prestranded filament, pitch, length of pre-stranded filament,

\footnotetext{
*Corresponding author’s e-mail: 773221288@qq.com
} 
spinning direction, sand blasting process[7]. Therefore, it is necessary to analyze the stress of tension clamp and select the diameter of pre-stranded filament.

\subsubsection{Stress analysis of tension clamp}

The stress analysis of tension clamp can be simplified as shown in Figure 1.

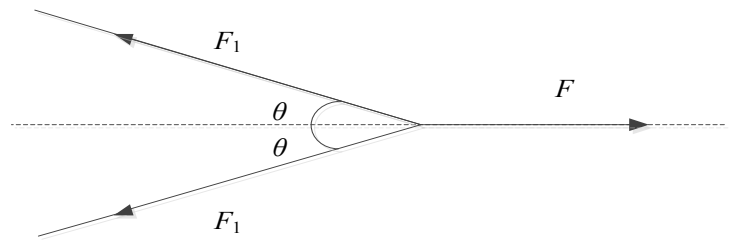

Figure 1. Stress diagram of tension clamp

According to GB/T 2314-2008[8], it is assumed that the calculated breaking force of conductor is $F_{0}$. The grip force required by the clamp is not less than $95 \%$ of the breaking force of the conductor, that is, the pulling force of a single clamp is:

$$
F_{1} \geq \frac{0.95 F_{0}}{2 \cos \theta}
$$

\subsubsection{Diameter Analysis of Pre-stranded Single Wire}

The tension and maximum allowable tension of unilateral pre-stranded wire are as follows:

$$
\begin{aligned}
& F_{1}=\frac{F}{2 \cos \theta} \\
& F=\frac{n \pi \mathrm{d}^{2} \sigma_{b}}{4 s}
\end{aligned}
$$

In order to meet the requirement of clamp strength, the maximum allowable pulling force must be greater than the pulling force of unilateral pre-stranded wire, thus determining the diameter of pre-stranded wire to meet the requirement.

\subsubsection{Forming aperture design (ID/OD)}

In order to generate compressive stress after wrapping the pre-stranded wire, the forming aperture must be smaller than the diameter of the suitable wire. This ID/OD value is generally between 0.72 and 0.94 according to domestic and foreign research and experiments, in this paper is 0.85 . Because the diameter of aluminum composite carbon fiber cores is much smaller than that of steel core, and if the pre-twisted tension is directly wound, the surface may be damaged by sand sticking during construction, so a pre-twisted protective line can be added to the outer layer of aluminum composite carbon fiber cores.

\subsubsection{Pitch design of pre-stranded wire}

The formula for calculating the intercept of pre-stranded wire is as follows:

$$
T=\pi(D+d) \operatorname{ctg} \theta
$$

Among them, $\mathrm{T}$ is the pitch of the pre-stranded wire, $\mathrm{D}$ is the diameter of the wire, $d$ is the diameter of the prestranded wire, and $\theta$ is the spiral angle of the prestranded wire forming. According to the previous production experience, $\theta=23.56$;

\subsection{Three Kinds of Pre-stranded Tension Clamp Structures Designed in This Paper}

According to the above analysis, this section designs three typical structures of tension-resistant clamp for different conductors. The basic structure includes ring insertion, carbon fiber core grip pre-winding, aluminum layer grip pre-winding and so on. The main difference is in carbon fiber composite core grip pre-winding. The structure schematic diagram is shown in Figure 2.

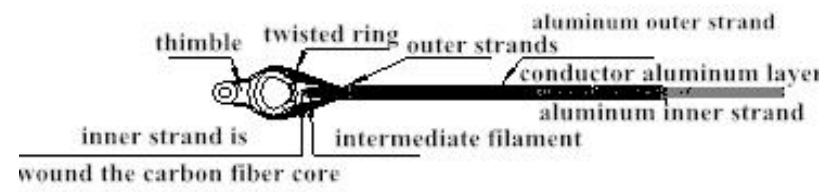

(a) Structure I

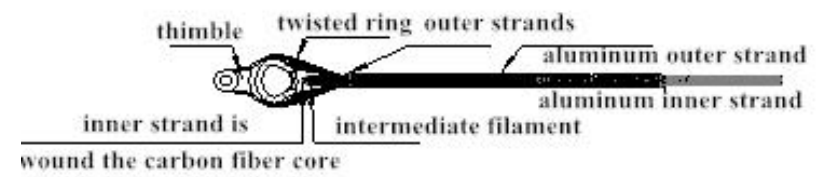

(b) Structure II

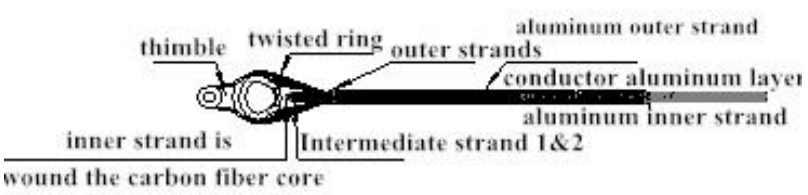

(c) Structure III

Figure 2. Structural design of pre-stranded tension clamp

As can be seen from the figure: the carbon fiber core grip pre-twisted wire in structure I and II is a three-layer twisted wire structure, which includes inner twisted wire, middle twisted wire and outer twisted wire.The carbon fiber core grip pre-strand in the third structure is a fourlayer structure, including inner strand, middle strand 1, middle strand 2 and outer strand. The grip pre-winding of the aluminum layer in the structure $\mathrm{I}$ is composed of an inner aluminum layer and an outer aluminum layer. Aluminum layer grip pre-winding part of structure II and III is single layer external winding.

\subsection{Examples of application of pre-stranded tension clamp}

Take the structure II as an example, the type of the aluminum composite carbon fiber cores is JLRX/T$450 / 50$, the diameter of conductor is $8.0 \mathrm{~mm}$ and the outer diameter of conductor is $26.1 \mathrm{~mm}$. as shown in Fig. 3. 


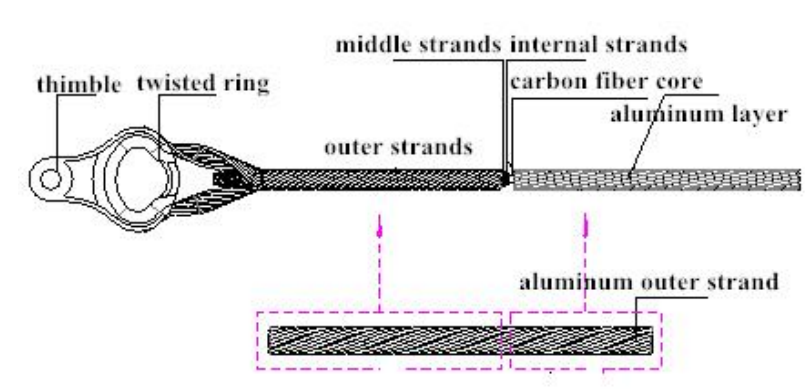

\section{Gripping Force Test of JLRX/T- 450/50 Tensile Wire}

In view of the structure of the pre-stranded tension clamp designed above, the strength and grip strength of the clamp are tested to verify whether the strength and grip strength of the clamp meet the design requirements. The required value of the grip strength of the tension clamp is shown in Table 1.

Figure 3. The examples of structure II

Table 1 Requirements for grip strength of tension wire

\begin{tabular}{c|c|c}
\hline Type & Rated Breaking Force/kN & GBT2314 Gripping Force Value/kN \\
\hline JLRX/T-450/50 & 150 & $\geq 142.5(150 \times 95 \%)$ \\
\hline
\end{tabular}

After the clamp is installed on the wire, the whole test is carried out. The distance between the two clamps is not less than 100 times of the diameter of the wire. The schematic diagram of the experimental components is shown in Fig. 4.

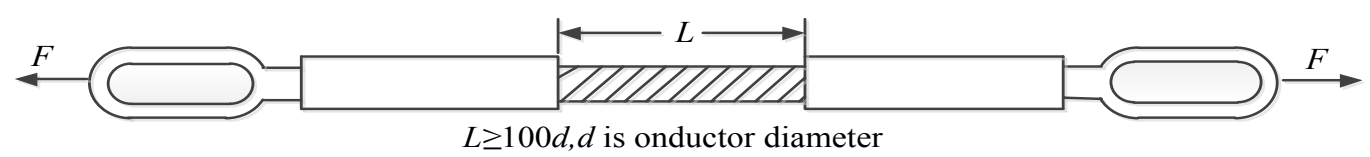

Figure 4. Schematic diagram of experimental components

In the test, if the conductor leaves the original scribed position but the load can continue to rise, it is not considered as sliding; when the load does not continue to increase and the conductor continues to displace, it is considered that the conductor slips. The experimental results are shown in Fig. 5.

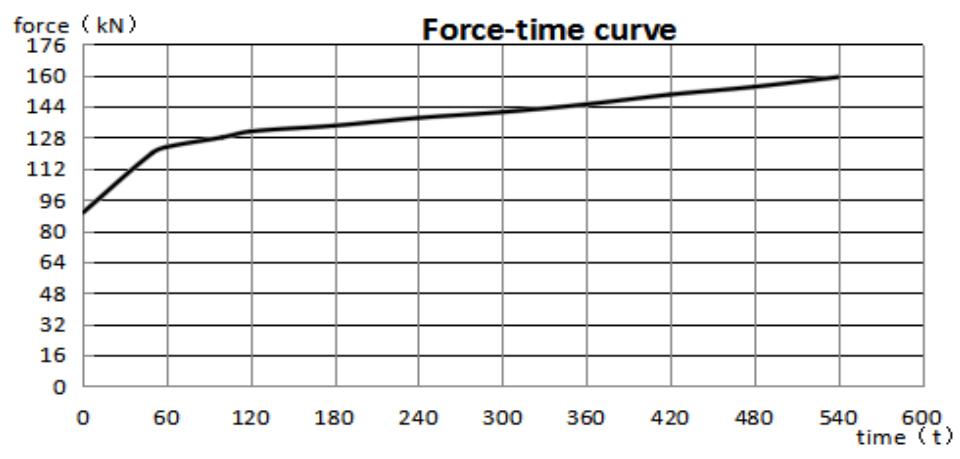

Figure 5. Experimental results

The experimental results show that: the calculated breaking force of the conductor with JLTX/T-450/50 wire type is $146.586 \mathrm{kN}$, which meets the requirements of GB/T 2314-2008. It shows that the structure of the tension clamp designed in this paper has good reliability.

\section{Installation Technology and Technical \& Economic Analysis of Pre-stranded Tension Clamp}

\subsection{Installation Process Analysis}

For aluminum composite carbon fiber cores, the pretwisted tension clamp has three advantages over the traditional wedge tension clamp in installation technology:Firstly, the traditional wedge-shaped tension clamp is very easy to damage the aluminum composite carbon fiber cores. Secondly, the installation of the traditional wedge tension clamp requires very high personnel. If operation is not in place, it will cause irreparable damage to the conductor. The installation of the pre-twisted tension clamp is not more difficult than the construction requirements of common wire fittings. Thirdly, the traditional wedge tension clamp also has requirements for the construction environment, bad weather will cause the problem of insufficient grip, and the construction conditions of pre-stranded tension clamp are basically the same as the installation requirements of common wire fittings. 


\subsection{Technical and Economic Analysis}

Taking JLRX/T-450/50 wire tension clamp as an example. According to the current market price, the comprehensive cost of traditional wedge-shaped selflocking tension clamp hardware is about 1500 yuan per set, and the comprehensive cost of the pre-twisted tension clamp hardware designed in this paper is about 600 yuan per set. According to the length of the transmission line $100 \mathrm{~km}$, the transmission line is erected according to "tension-drape-drape-tension" and the span is $400 \mathrm{~m}$. The number of tension-resistant fittings required is about 375 sets.In the construction process, considering the different construction technology, the pre-stranded tension clamp can be installed with only one person at a minimum, and the installation time of one set is about 10 minutes, while the traditional wedgeshaped self-locking tension clamp requires three people to install at the same time, and the installation time of one set is about 60 minutes. Labor costs are calculated at 50 yuan per person/hour, without considering unpredictable factors such as proficiency. The technical and economic results of the tension clamp designed in this paper are compared with those of the traditional wedge-shaped self-locking tension clamp, as shown in Table 2.

Table 2 Technical and economic comparison

\begin{tabular}{c|c|c}
\hline Items & Pre-stranded tension clamp & Wedge-shaped self-locking tension clamp \\
\hline Cost (yuan/set) & 600 & 1500 \\
\hline Labor cost & \multicolumn{2}{|c}{50 yuan/person/hour } \\
\hline Number (person) & 1 & 3 \\
\hline Time (min/set) & 10 & 60 \\
\hline Total cost (yuan) & 228125 & 581250 \\
\hline
\end{tabular}

Table 2 shows that the cost of pre-stranded tension clamp is about $40 \%$ of wedge-shaped self-locking tension clamp considering the construction technology and human cost, and the technical and economic effect is better than before.

\section{Conclusion}

Three kinds of pre-twisted tension clamps with different structures were designed to overcome the shortcomings of traditional metal fittings for carbon fiber composite core conductors, the experimental verification is also carried out. Finally, combined with the actual application situation, the technical and economic analyses are carried out. The results show that the pre-stranded tension clamp designed in this paper optimizes the construction process on the basis of fully guaranteeing the performance of the fittings, and is more economical than the existing technology in terms of technology and economy, which provides support for the popularization of carbon fiber composite core conductors.

\section{Acknowledgments}

This paper is supported by Science and Technology Project of State Grid Liaoning Electric Power Supply Co., Ltd.."Development and Application of High Conductivity and Heat Resistant Aluminum Alloy Conductor with Carbon Fiber Composite Core and Matched Pre-stranded Metal Fittings". Project number is 2017YF-8.

\section{References}

1. Ju Yanzhong, Li Qiuchen, Meng Yaman. Comparative Research on aluminum composite carbon fiber cores and Traditional Conductor [J]. East China Power, 2011 (7): 1191-1194..

2. Liu Guangzhong. Cause analysis of conductor shedding of wedge-shaped insulated tension clamp for $10 \mathrm{kV}$ transmission line $[\mathrm{J}]$. Electronic fabrication, 2017 (3-5): 62-63.

3. Li Guoran. Application of pre-stranded tension clamp in transmission line $[\mathrm{J}]$. Electrical and Electrical, 2010 (3): 52-54.

4. Dong Zhichun, He Fengsheng, Zhang Chunlei. Structural optimization of transmission line tension clamp [J]. Guangdong Electric Power, 2013 (12).

5. Liu Chun, Chen Hongdong, Ouyang Kejian. Fracture analysis of tension clamp of carbon fiber composite core conductor $[\mathrm{J}]$. China Electric Power, 48 (10).

6. Zou Guolin, Huaixian, Lv Quanggen. Development and Application of Chinese Overhead Transmission Line Fitting Technology $[\mathrm{J}]$. Power Engineering Technology, 2012, 31 (6): 82-84.

7. Tu Yongguo. Superiority and Installation Method of Pre-stranded Gold Fittings [J]. China Electronic Commerce, 2013 (9): 245-245.

8. GB/T 2314-2008 General Technical Conditions for Electric Power Fittings. 